\title{
Tratamento de Molusco Contagioso em crianças: uma revisão sistemática de literatura
}

\author{
Molluscum Contagiosum treatment in children: a systematic literature review \\ Tratamiento del molusco contagioso en niños: una revisión sistemática de la literatura
}

Diana de Oliveira Frauches, Carla Coradini de Mattos Siqueira, Thays Furtado Dias da Mata

\section{Resumo}

Objetivo: Molusco contagioso é uma infecção cutânea, causada pelo Molluscipox vírus. Diante da inexistência de um tratamento específico, esta pesquisa tem como objetivo verificar, segundo a literatura recente, os melhores métodos de tratamento em crianças imunocompetentes. Métodos: Revisão sistemática de literatura realizada no PubMed, bem como no Lilacs, Scielo, Medline e demais bases de dados abrangidas na Biblioteca Virtual em Saúde (BVS), com publicações de 2010 a 2016. Para evitar viés, foram utilizadas recomendações do PRISMA. Critérios do Oxford Centre for Evidence-Based Medicine e do sistema GRADE foram usados para classificação do nível de evidência dos estudos. Resultados: Curetagem, laser pulsado de contraste, crioterapia, exérese cirúrgica, $\mathrm{KOH} \mathrm{2,5 \% ,5 \%} \mathrm{e} \mathrm{10 \% ,}$ ácido tricloroacétrico, combinação de ácido lático e ácido salićlico, tretinoína 0,05\% apresentaram boa proporção de cura, facilidade de realização e tempo de resolução. Os métodos de injeção intralesional do antígeno da Cândida e da vacina MMR, dieta balanceada, óleo de Malaleuca alternifólia e Ingenol Mebutate 0,05\% mostraram-se promissores. Entretanto, cantaridina e imiquimod 5\% creme não foram recomendados. Conclusão: As evidências obtidas não permitem indicar um método preferencial. A escolha do método de tratamento deve ser individualizada. Recomenda-se realizar mais pesquisas voltadas para cultura do vírus.

\section{Abstract}

Objective: Molluscum contagiosum is a skin infection caused by the Molluscipox virus. Given the absence of a specific treatment, this study aims to verify according to recent literature the best methods for molluscum contagiosum treatment in immunocompetent children. Methods: This study is a systematic review conducted in PubMed, as well as Lilacs, Scielo, Medline and other data bases from the Biblioteca Virtual em Saúde (BVS), including publications from 2010 to 2016. The bias is avoided by using PRISMA recommendations . Criteria of the Oxford Center for Evidence-Based Medicine and the GRADE system were used to rank the level of evidence of the studies. Results: Curettage, pulsed dye laser, cryotherapy, surgical abscission, $\mathrm{KOH} 2.5 \%, 5 \%$ and $10 \%$, trichloroacetic acid, combination of lactic acid and salicylic acid and $0.05 \%$ tretinoin showed good performances regarding cure rate, ease of implementation and resolution time. Moreover, methods such intralesional injection of antigen Candida and the MMR vaccine, balanced diet, Malaleuca oil alternifolia and ingenol Mebutate $0.05 \%$ showed relevant results. However, the use of cantharidin and imiquimod $5 \%$ cream is not recommended. Conclusion: It is not possible to indicate the most eligible method based on the evidence found. Therefore, treatment should be individualized. Future researches about the virus culture are recommended.
Palavras-chave:

Molusco Contagioso

Terapêutica

Resultado do Tratamento

Keywords:

Molluscum Contagiosum

Therapeutics

Treatment Outcome
Como citar: Frauches DO, Siqueira CCM, Mata TFD. Tratamento de Molusco Contagioso em crianças: uma revisão sistemática de literatura. Rev Bras Med Fam Comunidade. 2017;12(39):1-12. http://dx.doi.org/10.5712/rbmfc12(39)1468
Fonte de financiamento: declaram não haver. Parecer CEP: não se aplica. Conflito de interesses: declaram não haver. Procedência e revisão por pares: revisado por pares. Recebido em: 15/02/2017. Aprovado em: 09/10/2017. 


\section{Resumen}

Objetivo: El molusco contagioso es una infección de la piel causada por el virus Molluscipox. Dada la ausencia de un tratamiento específico, la presente investigación tiene como objetivo verificar, de acuerdo con la literatura reciente, los mejores métodos de tratamiento en niños inmunocompetentes. Métodos: Revisión sistemática de la literatura realizada en el Pubmed, así como en Lilacs, Scielo, Medline y otras bases de datos en la Biblioteca Virtual de Salud (BVS), con publicaciones de 2010 a 2016. Para evitar sesgos, se utilizaron las recomendaciones del PRISMA. Se utilizaron los criterios del Centro de Oxford para la Medicina Basada en la Evidencia y el sistema GRADE para clasificar el nivel de evidencia de los estudios. Resultados: Curetaje, láser pulsado de contraste, crioterapia, exéresis quirúrgica, $\mathrm{KOH} 2,5 \%$, $5 \%$ y $10 \%$, ácido tricloroacétrico, la combinación de ácido láctico y ácido salicílico, tretinoína 0,05\% exhibieron buena tasa de curación, facilidad de realización y tiempo de resolución. Los métodos de inyección intralesional de antígenos de Cándida y la vacuna triple vírica, dieta equilibrada, aceite de alternifolia Malaleuca y ingenol mebutate $0,05 \%$ se muestran prometedores. Sin embargo, no se recomienda las cremas de cantaridina y imiquimod al $5 \%$. Conclusión: Las evidencias obtenidas no han elegido un método preferido. La elección del método de tratamiento debe ser individualizada. Se recomienda la realización de más investigaciones relcionadas con el cultivo del virus.

\section{Introdução}

Molusco Contagioso (MC) é uma infecção viral cutânea muito frequente, causada por um vírus com dupla cadeia de ácido desoxirribonucleico, pertencente à família dos poxvírus, gênero Molluscipox. ${ }^{1}$ Primeiramente descrito e nomeado por Bateman no início do século XIX. ${ }^{2}$

A doença se expressa por pápulas cor da pele, entre três a cinco milímetros de diâmetro, por vezes chegando a três centímetros ("molusco gigante"). As lesões normalmente apresentam uma umbilicação central de onde pode ser drenada uma substância branco-acinzentada extremamente virulenta. ${ }^{2}$

Os grupos mais acometidos são crianças e adolescentes ${ }^{2}$ entre 1 e 14 anos. ${ }^{3}$ Também são acometidos adultos sexualmente ativos e pessoas imunocomprometidas ou imunossuprimidas. Apresentação em crianças menores de um ano é rara, possivelmente devido à imunização materna. ${ }^{2}$

A transmissão se dá pelo uso de fômites contaminados, contato pele a pele e autoinoculação. ${ }^{4} \mathrm{O}$ tempo de incubação varia entre 14 dias a 6 meses. ${ }^{5}$ As lesões, em indivíduos imunocompetentes, tendem a ter resolução espontânea de meses a anos. ${ }^{6}$ É comum ocorrer recidiva. ${ }^{7}$

O diagnóstico é predominantemente clínico, porém lesões atípicas podem dificultar o fechamento do quadro. Neste caso, pode-se lançar mão de métodos como Gram, estudo histopatológico e microscopia eletrônica. ${ }^{2}$

Dentre as técnicas utilizadas para tratamento, existem métodos físicos, químicos e imunoterápicos, além da possibilidade de aguardar resolução espontânea do quadro, ${ }^{5}$ com regressão das lesões. Até o momento, não há terapêutica específica para tratamento de $\mathrm{MC}$, provavelmente por insucesso nas tentativas de cultivo do vírus em ambiente laboratorial. ${ }^{8}$

As lesões de MC podem levar a complicações, como: inflamação, prurido, eczemas, infecção bacteriana secundária e cicatrizes permanentes. ${ }^{9}$ Um estudo realizado por Lacour et al. ${ }^{10}$ relatou a ocorrência, em casos de MC, de abscessos causados por Staphylococcus lugdunensis.

Busca de informação sobre tratamento de $\mathrm{MC}$ na biblioteca da Colaboração Cochrane mostrou apenas uma revisão de literatura, realizada em 2009. ${ }^{5}$ Nela, van der Wouden et al. estudaram os efeitos das estratégias de tratamento de $\mathrm{MC}$ não genital em pacientes imunocompetentes. A revisão foi feita com base em 11 estudos que incluíam os efeitos de tratamentos tópicos, sistêmicos e homeopáticos. 
Segundo os autores, uma série de limitações desses estudos, como o fato de serem pequenos, com alguns pacientes abandonando o tratamento, entre outros, levou à consideração de que não foi identificada evidência suficiente para que se pudesse propor um tipo de intervenção para MC. Assim, foi sugerido que, até o surgimento novas evidências de um tratamento seguro e efetivo, profissionais médicos deveriam considerar a conduta expectante. ${ }^{5}$

O objetivo desta pesquisa é atualizar as informações sobre o tratamento de $M C$ em crianças imunocompetentes entre um e nove anos de idade, faixa etária na qual é alta a incidência ${ }^{3}$ e os hábitos e comportamentos favorecem a ocorrência de complicações infecciosas.

\section{Métodos}

Esta pesquisa consiste em revisão sistemática de literatura sobre tratamento de MC. A busca de referências foi realizada em junho de 2016 na base de dados PubMed, bem como no Medline, Lilacs, Scielo e demais bases abrangidas na Biblioteca Virtual em Saúde (BVS). Foram utilizados os descritores "Molusco Contagioso" e "Terapêutica" no título, resumo ou assunto e selecionadas publicações entre 2010 e 2016, em português, inglês e espanhol (critério de inclusão).

Das referências obtidas, excluíram-se aquelas que não discutissem tratamento ou não enfocassem em crianças entre um e nove anos de idade ou que abordassem apenas pacientes imunodeprimidos ou imunossuprimidos, além dos artigos aos quais não se obteve acesso ao texto completo.

O nível de evidência científica das referências foi classificado de duas formas. A primeira, de acordo com o grau de recomendação e os níveis de evidência estabelecidos pelo Nível de Evidência por Tipo de Estudo - Oxford Centre for Evidence-Based Medicine, utilizados pela Associação Médica Brasileira (AMB) no Projeto Diretrizes. ${ }^{11}$ Na segunda forma de classificação, foram observadas as recomendações estabelecidas pelo sistema Grading of Recommendations Assessment, Development and Evaluation (GRADE). ${ }^{12}$

Os métodos abordados nas referências foram comparados quanto à efetividade (maior ou menor potencial de cura das lesões), facilidade de realização (método tópico ou invasivo), custo, tempo de resolução das lesões e ocorrência ou não de recidiva, além da incidência e gravidade de efeitos colaterais.

Visando evitar risco de viés, foram adotados o fluxograma e o checklist da Preferred Reporting Items for Systematic Reviews and Meta-Analyses: The PRISMA Statement ${ }^{13}$ para condução da revisão. Esta recomendação em sua forma atual surgiu no Canadá em 2005, da revisão de um guia de recomendação de 1996 conhecido como Quality of Reporting of Meta-analyses (QUOROM).

\section{Resultados}

Foram identificados 113 artigos, dos quais foram eliminados os que não preencheram os critérios previamente estabelecidos, resultando em 22 artigos (Figura 1).

Ao avaliar as 22 publicações incluídas de acordo com o Nível de Evidência por Tipo de Estudo - Oxford Centre for Evidence-Based Medicine ${ }^{11} 11$ artigos se classificaram como grau de recomendação $B$, nível de confiança 2B; 2 como grau de recomendação $B$, nível de confiança $2 \mathrm{C} ; 5$ como grau de recomendação $C$, nível de confiança 5; e 4 como grau de recomendação $D$, nível de confiança 5. 


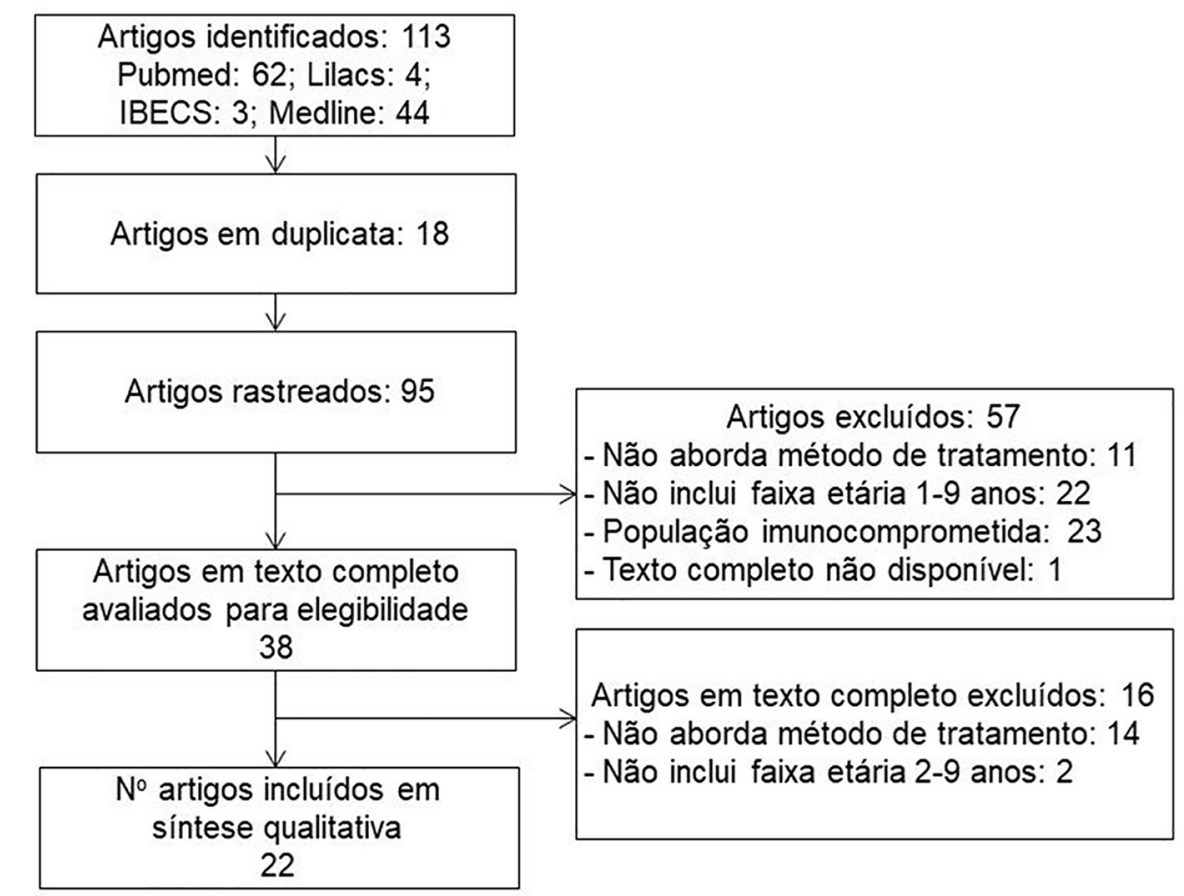

Figura 1. Fluxograma das fases de identificação, triagem e seleção de artigos sobre métodos de tratamento de $\mathrm{MC}$ em crianças imunocompetentes de dois a nove anos de idade.

A classificação dos níveis de evidência dos artigos utilizando o sistema GRADE ${ }^{12}$ apontou 10 artigos como nível de confiança baixo e 12 como nível de confiança muito baixo.

Características dos estudos relatados nos artigos e a classificação dos mesmos segundo o Oxford Centre for Evidence-Based Medicine e o sistema GRADE estão apresentadas no Quadro 1.

O tratamento de MC pode ser realizado através de métodos químicos, físicos e imunoterápicos.

A curetagem cirúrgica é um método amplamente utilizado, efetivo, com rápida resolução, porém pode apresentar efeitos colaterais como dor local, e não é indicada para lesões em áreas sensíveis como face. Schmitt e Marchiori ${ }^{14}$ mostraram que o uso da curetagem em até duas sessões teve uma proporção de cura de $26,6 \%$. Como efeito colateral houve relato de dor intensa e incômodo local. Machado et al. ${ }^{9}$ citaram como benefício a rapidez nos resultados e baixo número de complicações, em um grupo de estudo com seguimento de 90 dias. É um procedimento realizado em apenas um dia, no consultório, com anestésico local, apontando facilidade no tratamento de crianças.

A crioterapia local, outra técnica ainda muito utilizada, mostra-se bastante efetiva. $O$ estudo por Al-Mutairi et al. ${ }^{15}$ relatou proporção de cura de $100 \%$ com 1 sessão por semana até a cura ou em um máximo de 16 semanas. O tempo de resolução das lesões foi em média três a seis semanas, sendo a técnica mais barata que outros métodos, como o imiquimod. Pode haver recidiva dentro de até seis meses e levar a efeitos colaterais como alteração da pigmentação local, defeitos de cicatrização ou atrofia no local da aplicação.

Em relação ao Laser Pulsado de Contraste (LPC), Swanson e Canty, ${ }^{16}$ em sua atualização terapêutica com 20 crianças participantes, relatam que 16 apresentaram resolução completa das lesões com apenas duas sessões. Em revisão sistemática realizada por Griffith et al., ${ }^{17}$ vários autores relatam sucesso de 
Quadro 1. Características dos estudos e classificação do nível de evidência dos artigos.

\begin{tabular}{|c|c|c|c|}
\hline Artigo & Características do estudo & $\begin{array}{l}\text { Grau de recomendação } \\
\text { e nível de evidência }\end{array}$ & $\begin{array}{l}\text { Nível de } \\
\text { evidência GRADE }\end{array}$ \\
\hline Na et al., $2014 .^{6}$ & Relato de 2 casos, injeção intralesional da vacina MMR. & C 4 & Muito baixo. \\
\hline Machado et al., 2010.9 & $\begin{array}{l}\text { Ensaio clínico controlado randomizado, n=50, } 17 \text { com } \\
\mathrm{KOH} 10 \%, 16 \text { com ácido salicílico+ácido láctico e } 17 \mathrm{com} \\
\text { curetagem, seguimento de } 90 \text { dias. }\end{array}$ & B 2B & Baixo. \\
\hline Al-Mutairi et al., $2010 .^{15}$ & $\begin{array}{l}\text { Ensaio clínico controlado randomizado, duplo-cego, } \\
\mathrm{n}=74,37 \text { com imiquimod creme } 5 \% \text { e } 37 \text { com crioterapia, } \\
\text { seguimento de } 6 \text { meses. }\end{array}$ & B $2 B$ & Baixo. \\
\hline Griffith et al., $2014 .{ }^{17}$ & $\begin{array}{l}\text { Revisão sistemática de literatura, laser pulsado de } \\
\text { contraste. }\end{array}$ & B 2C & Muito baixo. \\
\hline Falzon et al., $2015 .^{18}$ & Relato de 1 caso, exérese cirúrgica. & C 4 & Muito baixo. \\
\hline Katz e Swetman, 2013. ${ }^{19}$ & Opinião de especialista, imiquimod creme $5 \%$. & D 5 & Muito baixo. \\
\hline Katz, 2015. ${ }^{20}$ & Opinião de especialista, imiquimod creme $5 \%$. & D 5 & Muito baixo. \\
\hline Smith et al., 2013. ${ }^{22}$ & Relato de 2 casos, imiquimod creme $5 \%$. & C 4 & Muito baixo. \\
\hline Coloe Dosal et al., $2014 .{ }^{23}$ & $\begin{array}{l}\text { Ensaio clínico controlado randomizado, n=29, } 13 \text { com } \\
\text { cantaridina e } 16 \text { com placebo, seguimento de } 2 \text { meses. }\end{array}$ & B 2B & Baixo. \\
\hline Moye et al., 2013. ${ }^{24}$ & Opinião de especialista, cantaridina. & D 5 & Muito baixo. \\
\hline Rajouria et al., $2011 .{ }^{29}$ & $\begin{array}{l}\text { Ensaio clínico controlado randomizado, } \mathrm{n}=50,25 \\
\text { com } \mathrm{KOH} 5 \% \text { e } 25 \text { com creme de tretinoína } 0,05 \% \text {, } \\
\text { seguimento de } 4 \text { semanas. }\end{array}$ & B 2B & Baixo. \\
\hline Enns e Evans, $2013 .{ }^{30}$ & $\begin{array}{l}\text { Ensaio clínico não controlado, n=29, injeção intralesional } \\
\text { do antígeno da Cândida, } 15 \text { meses de seguimento. }\end{array}$ & B 2B & Baixo. \\
\hline van der Gaag, 2013. ${ }^{31}$ & Ensaio clínico não controlado, n=7, dieta balanceada. & B 2B & Muito baixo. \\
\hline Markum e Baillie, $2012 .{ }^{32}$ & $\begin{array}{l}\text { Ensaio clínico controlado randomizado, n=53, } 19 \text { com } \\
\text { óleo de Malaleuca alternifolia+óleo de canola+iodo, } 18 \\
\text { com óleo de Malaleuca alternifolia+óleo de canola e } 16 \\
\text { com iodo, seguimento de } 30 \text { dias. }\end{array}$ & B 2B & Baixo. \\
\hline Javed e Tyring, $2014 .^{33}$ & Relato de 1 caso, ingenol mebutate creme. & C 4 & Muito baixo. \\
\hline
\end{tabular}


tratamento, com a proporção de cura entre $67 \%$ a 99\%, além de fácil e rápida aplicação, que dura cerca de dois a três segundos. Não houve recidivas e como efeitos colaterais foram notados púrpuras e pigmentação transitória da pele durante o procedimento.

Um relato de caso por Falzon et al. ${ }^{18}$ traz como opção de tratamento a exérese cirúrgica, a qual levou à resolução do caso em questão. Apesar disso, o artigo não apresenta dados sobre a facilidade do uso, duração do tratamento, recidivas ou efeitos colaterais.

Dentre os métodos químicos, um dos mais utilizados e controversos, é o uso tópico de imiquimod $5 \%$ creme. Em um artigo de Katz e Swetman ${ }^{19}$ é relatado um estudo envolvendo 323 crianças, com eliminação completa das lesões em 18 semanas em $24 \%$ dos doentes tratados com imiquimod e em $26 \%$ dos tratados com placebo. Katz ${ }^{20}$ descreve, em um estudo que envolveu 379 crianças, que a eliminação completa ocorreu em $24 \%$ e $28 \%$, respectivamente. Além disso, as crianças apresentaram maior propensão a reações no sítio de aplicação, otite média, conjuntivite, leucopenia, e linfadenopatia. Ainda de acordo com Katz e Swetman, ${ }^{19}$ a agência Food and Drug Administration (FDA) afirmou em 2007: "Eficácia não demonstrada para MC em crianças com idade entre 2 a 12 anos", e a aplicação de imiquimod a pelo menos $10 \%$ da área de superfície corporal levou a leucopenia ou neutropenia em $40 \%$ e $25 \%$ dos pacientes, respectivamente.

Swanson e Canty ${ }^{16}$ comparam dois estudos sobre o uso do imiquimod. O primeiro ${ }^{21}$ relata certa melhora das lesões após quatro semanas, porém nenhum paciente apresentou resolução completa das mesmas, e o segundo ${ }^{15}$ envolvendo 37 pacientes dos quais 22 apresentaram resolução completa das lesões com seis semanas, somando-se 34 com resolução completa ao final de 12 semanas de tratamento com imiquimod tópico.

Os pesquisadores ${ }^{15,21}$ concordam que o método é fácil de ser realizado, apesar de ser um medicamento de custo elevado e que necessita de várias aplicações, podendo ser aplicado em casa e utilizado em regiões sensíveis como face e genitália, providenciando um resultado mais estético. Não houve recidivas, mas foram descritos quadros de reação adversa no local da aplicação - hipopigmentação local, otite média, conjuntivite, leucopenia, linfadenopatia, leucopenia e neutropenia, com efeitos podendo aparecer após a primeira aplicação.

Em Smith et al., ${ }^{22}$ o uso de imiquimod 5\% creme foi correlacionado com o aparecimento de reação psoríase-like em uma criança de sete anos, após três semanas de uso da medicação, e de ulcerações orais em uma criança de nove anos, após nove dias de uso do medicamento no mento. Em Al-Mutairi et al., ${ }^{15}$ as crianças que foram tratadas com imiquimod creme a $5 \%$ apresentaram proporção de cura de $91,8 \%$ em 6 a 12 semanas, sem ocorrência de recidivas. Os efeitos colaterais não foram especificados.

O uso da cantaridina também é um método amplamente utilizado na medicina para o tratamento do MC. Swanson e Canty ${ }^{16}$ descreveram dor local e erupções cutâneas bolhosas graves. Em Coloe et al., ${ }^{23}$ cantaridina e placebo não se mostraram diferentes com respeito à proporção de alteração na resposta média, que não foi estatisticamente significativa. A proporção estimada foi de $32 \%$ menos lesões com cantaridina, enquanto de $21 \%$ menos com placebo. Os autores afirmam que os efeitos adversos são mínimos e que não houve boa resposta em dois meses.

Moye et al. ${ }^{7}$ realizaram revisão retrospectiva de prontuários de um dermatologista pediátrico, concluindo pela fácil utilização, tratamento rápido e ausência de dor na aplicação, sendo aprovado por pais 
e dermatologistas. Entretanto, os autores não citam proporção de cura, recidiva ou tempo de tratamento. Os efeitos colaterais encontrados foram dor, bolhas, prurido, infecção, irritação, hemorragia.

O artigo de Moye et al. ${ }^{24}$ cita que $92 \%$ dos dermatologistas pediátricos demonstraram satisfação com a cantaridina, por possuir tempo de tratamento rápido, baixa incidência de dor no momento da aplicação e não causar sangramento. Por outro lado, apresentou como desvantagem a utilização exclusiva ao consultório, por ser tóxica. Os efeitos adversos encontrados foram dor, bolhas, prurido, alterações pigmentares e infecção secundária.

O ácido tricloroacético em formulações de $20 \%$ ou 35\%, segundo Swanson e Canty ${ }^{16}$ é aplicado no consultório, com um cotonete, sobre a lesão de $\mathrm{MC}$, até que surja uma camada de congelação sobre o local. Pode levar a um pequeno eritema local, mas apresenta bons resultados curativos.

O uso de solução aquosa de hidróxido de potássio $(\mathrm{KOH})$ 10\% se mostrou extremamente custo-efetivo. Método barato, menos agressivo, podendo ser aplicado em domicílio. Em um estudo, Can et al. ${ }^{25}$ obtiveram $100 \%$ de cura ao final de quatro semanas de tratamento, sem que houvesse recidivas em um período de follow-up de três meses. No entanto, efeitos colaterais foram relatados: hipopigmentação local, queimação local severa, infecção secundária e surgimento de cicatriz hipertrófica nos locais aplicados.

Em estudo de Köse et al. ${ }^{26} \mathrm{com} 26$ pacientes, 12 foram tratados com uma solução de $\mathrm{KOH}$ a $10 \%$ e 14 com combinação de ácido salicílico e láctico durante 6 semanas. Ao final, 83,3\% do grupo de $\mathrm{KOH}$ demonstraram remissão completa e $16,7 \%$ deles apresentaram remissão parcial, mas quatro pacientes (33\%) desenvolveram novas lesões durante o estudo. Todos no grupo de combinação de ácido salicílico e láctico (100\%) demonstraram a remissão completa ao final de seis semanas, com cinco pacientes (35\%) adquirindo novas lesões durante o estudo. Observou-se facilidade de realização, sendo que solução de $\mathrm{KOH} 10 \%$ e combinação de ácido salicílico e láctico foram igualmente eficazes no tratamento de lesões de MC. Como efeitos adversos com uso de KOH 10\% foram observadas infecção, hipopigmentação e ardência. A combinação de ácido salicílico e láctico pode apresentar eritema, descamação e ulceração.

O estudo de Machado et al. ${ }^{9}$ também comparou os resultados do uso de $\mathrm{KOH} 10 \%$, combinação de ácido salicílico e láctico e curetagem, sendo a curetagem mais rápida, porém igualmente eficaz à combinação de ácido láctico e salicílico. A conclusão sobre a eficácia de $\mathrm{KOH} 10 \%$ comparado a ácido salicílico combinado a ácido lático foi a mesma que em Köse et al. ${ }^{26}$

Stephenson et al. ${ }^{27}$ relataram eficácia parecida entre as soluções e fácil aplicação, apresentando resolução de uma a cinco semanas, podendo causar ardor, eritema, prurido, dor, formação de crostas, hiperpigmentação e erosão como efeitos adversos. O estudo de Uçmak et al. ${ }^{28}$ também mostrou semelhança na efetividade, porém relatou que $\mathrm{KOH}$ a $2,5 \%$ apresentou melhores resultados em face. Os resultados foram de $44 \%$ de cura após 60 dias e a ardência foi o efeito colateral mais comum.

Outro método citado nos artigos foi a tretinoína creme 0,05\%, que, comparada ao $\mathrm{KOH} 5 \%$, apresentou eficácia semelhante, porém com demora na resolução, de acordo com Rajouria et al. ${ }^{29}$

A injeção intralesional do antígeno da Cândida visa a manipulação do sistema imune a fim de atingir uma resposta imune específica, tendo sido realizados estudos prévios envolvendo injeções intralesionais de Cândida, caxumba e tricophyton em verrugas - utilizados devido as altas taxas de resposta imune corporais a esses patógenos na população em geral. ${ }^{30}$ 
O mecanismo exato de ação deste método ainda não é claro. O tratamento é realizado com intervalos de quatro semanas entre as aplicações, com aplicações de $0,3 \mathrm{ml}$ do antígeno em uma das pápulas de MC, podendo ser abordadas até 3 pápulas se as lesões forem muito pequenas. Enns e Evans, ${ }^{30}$ de um total de 29 pacientes, observaram 27 com melhora total ou parcial, com apenas 2 pacientes não apresentando melhora após cerca de seis sessões. Outro estudo apresentado por Swanson e Canty ${ }^{16}$ em sua revisão foi realizado com 47 pacientes. Destes, 25 cumpriram o follow-up, dos quais 21 apresentaram resolução total ou parcial e quatro não tiveram melhora após quatro sessões. O único efeito adverso apresentado foi desconforto local no momento da injeção.

A imunoterapia com injeção intralesional da vacina MMR é ainda muito recente. Sua realização se dá da mesma maneira que a terapia com o antígeno da Cândida. Na et al. ${ }^{6}$ trazem dois relatos de caso, de uma criança de quatro anos e de uma criança de um ano, que apresentavam lesões de MC em vários sítios corporais, obtendo remissão completa após quatro e duas sessões respectivamente, sem a ocorrência de recidivas ou efeitos adversos. De acordo com os autores, esta pode ser uma técnica mais segura, barata, com menos efeitos colaterais e uma excelente opção à métodos abrasivos que tendem a ser menos aceitos por crianças menores.

Um método interessante apresentado por van der Gaag $^{31}$ é a dieta balanceada, fornecendo à criança alimentos ricos em ferro, zinco, vitaminas $A, C$ e E várias vezes na semana, a fim de melhorar sua imunidade natural e combater o vírus do MC sistemicamente. Essa técnica apresentou uma proporção de cura de $86 \%$ dos pacientes em 5 meses, com os $24 \%$ restantes apresentando $50 \%$ de cura das lesões na mesma época. Nenhum efeito colateral foi relatado.

Pode-se citar também o uso de óleo de Malaleuca alternifolia combinado a iodo, relatado por Markum e Baillie, ${ }^{32}$ que mostrou cura em 48 das 53 crianças avaliadas, após trinta dias. Os efeitos colaterais se restringiram a região avermelhada no local de aplicação.

Javed e Tyring ${ }^{33}$ fizeram uso de Ingenol Mebutate 0,05\% durante 6 dias em uma criança de 4 anos, observando melhora completa da lesão, sem recidiva em 30 dias. Os efeitos adversos foram vermelhidão no sítio de aplicação nos três primeiros dias.

\section{Discussão}

A curetagem foi apresentada ${ }^{9,14}$ como uma boa opção, com alto potencial de cura, rápida, trazendo poucos efeitos colaterais - como dor e incômodo locais. Um ponto negativo de seu uso são as lesões muito numerosas ou que se encontram em áreas mais sensíveis, como face e genitália.

A crioterapia local mostrou ${ }^{15}$ uma proporção de cura extremamente alta, atingindo $100 \%$. No entanto, houve recidiva das lesões e efeitos colaterais como alteração de pigmentação local, formação de cicatriz e atrofia. Trata-se de um bom método para resolução rápida, desde que o paciente seja esclarecido dos possíveis riscos de comprometimento estético.

A terapia com LPC ${ }^{16,17}$ constitui uma das melhores para o tratamento, com excelente potencial de cura. Não apresentou recidivas e os efeitos colaterais foram inexistentes ou transitórios durante o procedimento. A maior problemática é o elevado custo da máquina, dificultando sua aquisição e encarecendo o tratamento. 
A exérese cirúrgica mostrou-se eficaz. ${ }^{18}$ No entanto, apesar da cirurgia dermatológica ser extremamente segura e com baixo risco de complicações, os procedimentos cirúrgicos estão sujeitos a infecções, hemorragias, hematomas ou alterações da pressão arterial. ${ }^{34}$

O imiquimod $5 \%$ creme, alvo de grandes discussões e divergências literárias, ${ }^{15,16,19-22}$ não é uma técnica boa ao serem comparados riscos e benefícios, além de ter custo elevado. Apesar de se mostrar efetivo, de fácil aplicação, bons resultados estéticos e não serem comuns recidivas, não resultou grande superioridade em relação ao placebo, que justificasse expor o paciente aos riscos de efeitos colaterais.

A cantaridina não se mostrou eficaz,,16,23,24 apresentando proporção de cura baixa quando comparada ao placebo, recidivas e, por vezes, efeitos colaterais. Acredita-se que sua larga utilização é devido à fácil utilização, falta de dor à aplicação e resolução rápida em certo número de casos, sendo aprovado por pais e dermatologistas.

O ácido tricloroacético a $20 \%$ ou $35 \%$ é um método efetivo e simples, resultando em poucos efeitos colaterais e boa proporção de cura. ${ }^{16}$

O KOH em suas formulações 2,5\% (mais indicado para lesões em face), 5\% e 10\%, ${ }^{25,27-29}$ revelou-se, no geral, uma excelente técnica, de baixo custo, sem recidivas, com alta proporção de cura, resolução rápida das lesões, fácil aplicação, apresentando alguns efeitos colaterais locais. Todas as formulações se mostraram igualmente efetivas. Quando comparada sua eficácia à da formulação de ácido lático com ácido salicílico ${ }^{26}$ e à curetagem cirúrgica e à formulação ácida, ${ }^{9}$ a formulação apresentou velocidade de resolução das lesões superior à do $\mathrm{KOH}$, porém mesma efetividade, podendo apresentar efeitos colaterais. A curetagem mostrou-se superior a ambos em tempo de resolução, porém igualmente eficaz. Em comparação com a tretinoína $0,05 \%$, o $\mathrm{KOH} 5 \%$ mostrou ação mais rápida sobre as lesões. ${ }^{29}$

Os métodos imunoterápicos como a injeção intralesional do antígeno da Cândida ${ }^{3,30}$ e a injeção intralesional da vacina $\mathrm{MMR}^{6}$ mostraram um grande potencial. São extremamente eficazes, não apresentaram recidivas ou efeitos colaterais, a resolução das lesões foi rápida, de baixo custo, com abordagem não extensa no paciente, sendo necessária a aplicação em no máximo três lesões. No entanto, mais estudos sobre essas terapias fazem-se necessários para que se possa chegar a conclusões mais embasadas.

A dieta balanceada, ${ }^{31}$ o uso do óleo de Malaleuca alternifolia combinado a iodo ${ }^{32}$ e o uso de Ingenol Mebutate $0,05 \%{ }^{33}$ apresentaram alta proporção de cura. No entanto, estudos mais aprofundados sobre estas metodologias devem ser conduzidos a fim de avaliar a viabilidade de seu uso na prática clínica.

Nesta revisão, assim como na de $2009,{ }^{5}$ os estudos abordados foram considerados insuficientes para definir qual o melhor método de tratamento para MC. Em relação ao imiquimod creme 5\%, esta revisão encontrou resultados diferentes daqueles trazidos pela revisão Cochrane. Em van der Wouden et al., ${ }^{5}$ o imiquimod creme $5 \%$ foi relatado como tendo efeito superior ao placebo, mesmo que em um estudo considerado não estatisticamente efetivo, enquanto que nesta revisão, em todos os estudos recentes, o imiquimod não apresentou efeito superior ao placebo quanto à proporção de cura e tempo de resolução. 


\section{Conclusão}

Curetagem, LPC, crioterapia, exérese cirúrgica, $\mathrm{KOH} 2,5 \%, 5 \%$ e 10\%, ácido tricloroacético, combinação de ácido lático e ácido salicílico e tretinoína 0,05\% apresentaram bons resultados em relação à proporção de cura, facilidade de realização e tempo de resolução.

Os métodos de injeção intralesional do antígeno da Cândida e dieta balanceada mostraram-se promissores, mas é aconselhável que sejam estudados mais a fundo.

A injeção intralesional da vacina MMR, óleo de Malaleuca alternifólia e Ingenol Mebutate 0,05\% também necessitam de mais estudos com a finalidade de alcançar resultados mais concretos.

Cantaridina e imiquimod $5 \%$ creme não são recomendados como tratamento de escolha.

Por fim, a conduta expectante ainda deve ser uma opção considerada em crianças imunocompetentes, destacando-se que a escolha do método de tratamento deverá ser individualizada para cada paciente.

Ainda se fazem necessários estudos melhor concebidos, prospectivos, randomizados, de duração mais prolongada, com maior número de pacientes, controlados, sobre opções convencionais de tratamento comparadas a placebo. Da mesma forma, são importantes estudos laboratoriais visando buscar técnicas para cultura do vírus do MC e o desenvolvimento de um tratamento sistêmico eficaz contra a infecção, e não apenas das lesões.

\section{Referências}

1. Gaspar MA, Pinherio AF, Sanches A. Molusco contagioso - intervenção terapêutica na idade pediátrica. Revisão Baseada na Evidência. Rev Port Med Geral Fam [online]. 2012;28(6):418-26. http://www.scielo.mec.pt/pdf/rpmgf/v28n6/v28n6a06.pdf

2. Maluki AH, Kadhum QJ. Treatment of Molluscum Contagiosum by Potassium Hydroxide Solution $20 \%$ with and Without Pricking and By Pricking Alone: A Comparative Study with Review of Literature. Int J Dermatol Clin Res [online]. 2015;1(2):31-42. http://www.peertechz. com/Dermatology-Clinical-Research/pdf/JDCR-1-111.pdf. DOI: http://dx.doi.org/10.17352/2455-8605.000011

3. Olsen JR, Gallacher J, Finlay AY, Piguet V, Francis NA. Time to resolution and effect on quality of life of molluscum contagiosum in children in the UK: a prospective community cohort study. Lancet Infect Dis [online]. 2015;15(2):190-5. DOI: http://dx.doi.org/10.1016/ S1473-3099(14)71053-9

4. Ianhez M, Cestari SCP, Enokihara MY, Sieze MBPM. Padrões dermatoscópicos do molusco contagioso: estudo de 211 lesões confirmadas por exame histopatológico. An Bras Dermatol [online]. 2011;86(1):74-9. DOI: http://dx.doi.org/10.1590/S0365-05962011000100009

5. van der Wouden JC, van der Sande R, van Suijlekom-Smit LW, Berger M, Butler CC, Koning S. Interventions for cutaneous molluscum contagiosum. Cochrane Database Syst Rev [online]. 2009;(4):CD004767. DOI: http://dx.doi.org/10.1002/14651858.CD004767.pub3

6. $\mathrm{Na} \mathrm{CH}$, Kim DJ, Kim MS, Kim JK, Shin BS. Successful treatment of molluscum contagiosum with intralesional immunotherapy by measles, mumps, and rubella vaccine: a report of two cases. Dermatol Ther [online]. 2014;27(6):373-6. DOI: http://dx.doi.org/10.1111/dth.12158

7. Moye VA, Cathcart S, Morrell DS. Safety of cantharidin: a retrospective review of cantharidin treatment in 405 children with molluscum contagiosum. Pediatr Dermatol [online]. 2014;31(4):450-4. DOI: http://dx.doi.org/10.1111/pde.12276

8. Guan H, Nuth M, Zhukovskaya N, Saw YL, Bell E, Isaacs SN, et al. A novel target and approach for identifying antivirals against molluscum contagiosum virus. Antimicrob Agents Chemother [online]. 2014;58(12):7383-9. DOI: http://dx.doi.org/10.1128/AAC.03660-14

9. Machado RB, Leal TF, Bonfá R, Werlang ME, Weber MB. Molusco contagioso em crianças: tratamentos comparativos. Surg Cosmet Dermatol [online]. 2010;2(4):272-5. http://www.surgicalcosmetic.org.br/detalhe-artigo/94 
10. Lacour M, Posfay-Barbe KM, La Scala GC. Staphylococcus lugdunensis abscesses complicating molluscum contagiosum in two children. Pediatr Dermatol [online]. 2015;32(2):289-91. DOI: http://dx.doi.org/10.1111/pde.12483

11. Cerri GG, Jatene FB, Nobre MR, Bernardo WM. Introdução. In: Amaral JL, Andrade EO, eds. Projeto Diretrizes [online]. 2008. http:// diretrizes.amb.org.br/_BibliotecaAntiga/texto_introdutorio.pdf

12. Brasil. Ministério da Saúde. Secretaria de Ciência, Tecnologia e Insumos Estratégicos. Diretrizes metodológicas: Sistema GRADE Manual de graduação da qualidade da evidência e força de recomendação para tomada de decisão em saúde [online]. 2014. [acesso 2017 Nov 7]. Disponível em: http://bvsms.saude.gov.br/bvs/publicacoes/diretrizes_metodologicas_sistema_grade.pdf

13. Moher D, Liberati A, Tetzlaff J, Altman DG; PRISMA Group. Preferred reporting items for systematic reviews and meta-analyses: the PRISMA statement. PLoS Med [online]. 2009;6(7):e1000097. DOI: http://dx.doi.org/10.1371/journal.pmed.1000097

14. Schmitt JV, Marchiori HZ. Baixa eficácia do uso de solução de hidróxido de potássio a $5 \%$ por 28 dias no tratamento de molusco contagioso. Surg Cosmet Dermatol [online]. 2011;3(4):368-9. http://www.surgicalcosmetic.org.br/detalhe-artigo/177

15. Al-Mutairi N, Al-Doukhi A, Al-Farag S, Al-Haddad A. Comparative study on the efficacy, safety, and acceptability of imiquimod $5 \%$ cream versus cryotherapy for molluscum contagiosum in children. Pediatr Dermatol [online]. 2010;27(4):388-94. DOI: http://dx. doi.org/10.1111/ j.1525-1470.2009.00974.x

16. Swanson A, Canty K. Common pediatric skin conditions with protracted courses: a therapeutic update. Dermatol Clin [online]. 2013;31(2):239-49. http://www.ncbi.nlm.nih.gov/pubmed/23557653. DOI: http://dx.doi.org/10.1016/j.det.2012.12.003

17. Griffith RD, Yazdani Abyaneh MA, Falto-Aizpurua L, Nouri K. Pulsed dye laser therapy for molluscum contagiosum: a systematic review. J Drugs Dermatol [online]. 2014;13(11):1349-52. http://www.ncbi.nlm.nih.gov/pubmed/25607701

18. Falzon K, Scotcher S, Parulekar M. Primary Epibulbar Molluscum Contagiosum in an Immunocompetent Child. J Pediatr [online]. 2015;167(4):936. DOI: http://dx.doi.org/10.1016/j.jpeds.2015.06.071

19. Katz KA, Swetman GL. Imiquimod, molluscum, and the need for a better "best pharmaceuticals for children" act. Pediatrics [online]. 2013;132(1):1-3. DOI: http://dx.doi.org/10.1542/peds.2013-0116

20. Katz KA. Dermatologists, imiquimod, and treatment of molluscum contagiosum in children: righting wrongs. JAMA Dermatol [online]. 2015;151(2):125-6. DOI: http://dx.doi.org/10.1001/jamadermatol.2014.3335

21. Myhre PE, Levy ML, Eichenfield LF, Kolb VB, Fielder SL, Meng TC. Pharmacokinetics and safety of imiquimod $5 \%$ cream in the treatment of molluscum contagiosum in children. Pediatr Dermatol [online].2008;25(1):88-95. DOI: http://dx.doi.org/10.1111/j.1525-1470.2007.00590.x

22. Smith WA, Siegel D, Lyon VB, Holland KE. Psoriasiform eruption and oral ulcerations as adverse effects of topical $5 \%$ imiquimod treatment in children: a report of four cases. Pediatr Dermatol [online].2013;30(6):e157-60. DOI: http://dx.doi.org/10.1111/j.1525-1470.2012.01780.x

23. Coloe Dosal J, Stewart PW, Lin JA, Williams CS, Morrell DS. Cantharidin for the treatment of molluscum contagiosum: a prospective, doubleblinded, placebo-controlled trial. Pediatr Dermatol [online]. 2014;31(4):440-9. DOI: http://dx.doi.org/10.1111/j.1525-1470.2012.01810.x

24. Moye V, Cathcart S, Burkhart CN, Morrell DS. Beetle juice: a guide for the use of cantharidin in the treatment of molluscum contagiosum. Dermatol Ther [online]. 2013;26(6):445-51. DOI: http://dx.doi.org/10.1111/dth.12105

25. Can B, Topaloğlu F, Kavala M, Turkoglu Z, Zindancı I, Sudogan S. Treatment of pediatric molluscum contagiosum with 10\% potassium hydroxide solution. J Dermatolog Treat [online]. 2014;25(3):246-8. DOI: http://dx.doi.org/10.3109/09546634.2012.697988

26. Köse O, Özmen I, Arca E. An open, comparative study of $10 \%$ potassium hydroxide solution versus salicylic and lactic acid combination in the treatment of molluscum contagiosum in children. J Dermatolog Treat [online]. 2013;24(4):300-4. DOI: http://dx.doi.org/10.3109/09 546634.2011 .649690

27. Potassium hydroxide 5\% for the treatment of molluscum contagiosum. Drug Ther Bull [online]. 2014;52(10):118-20. http://www.ncbi.nlm. nih.gov/pubmed/25298120

28. Uçmak D, Akkurt MZ, Kacar SD, Sula B, Arica M. Comparative study of $5 \%$ and $2.5 \%$ potassium hydroxide solution for molluscum contagiosum in children. Cutan Ocul Toxicol [online]. 2014;33(1):54-9. DOI: http://dx.doi.org/10.3109/15569527.2013.796479 
29. Rajouria EA, Amatya A, Karn D. Comparative study of $5 \%$ potassium hydroxide solution versus $0.05 \%$ tretinoin cream for Molluscum Contagiosum in children. Kathmandu Univ Med J (KUMJ) [online]. 2011;9(36):291-4. DOI: http://dx.doi.org/10.3126/kumj.v9i4.6347

30. Enns LL, Evans MS. Intralesional immunotherapy with Candida antigen for the treatment of molluscum contagiosum in children. Pediatr Dermatol [online]. 2011;28(3):254-8. DOI: http://dx.doi.org/10.1111/j.1525-1470.2011.01492.x

31. van der Gaag E. Molluscum contagiosum clearance following a dietary change. Cutis [online]. 2013;92(4):E3-4. http://www.ncbi.nIm.nih. gov/pubmed/24195099

32. Markum E, Baillie J. Combination of essential oil of Melaleuca alternifolia and iodine in the treatment of molluscum contagiosum in children. J Drugs Dermatol [online]. 2012;11(3):349-54. http://www.ncbi.nlm.nih.gov/pubmed/22395586

33. Javed S, Tyring SK. Treatment of molluscum contagiosum with ingenol mebutate. J Am Acad Dermatol [online]. 2014;70(5):105. DOI: http://dx.doi.org/10.1016/j.jaad.2013.11.046

34. Pimentel ERA, Oliveira JP, Bloch LD, Niwa ABM. Risco de complicações durante a cirurgia dermatológica: protocolo das exéreses em fuso. An Bras Dermatol [online]. 2005;80(5):493-8. DOI: http://dx.doi.org/10.1590/S0365-05962005000600007

Escola Superior de Ciências da Saúde Santa Casa de Misericórdia de Vitória (EMESCAM). Vitória, ES, Brasil. diana.frauches@emescam.br (Autora correspondente); carlacoradinim@gmail.com; thaysfdmata@gmail.com 